\title{
Immagini e strutture in un racconto della Deledda
}

\section{Giuliana Sanguinetti Katz}

In questa nota illustrero il modo in cui la Deledda rappresenta il mondo della famiglia, tramite l'esame dettagliato di un suo racconto particolarmente significativo, intitolato "Battesimi", apparso nella terza pagina del Corriere della sera del 6 aprile del 1928 e ripubblicato nel 1930 nella raccolta di novelle Casa del poeta (71-78). ${ }^{1}$ Come ha ben dimostrato il Piromalli (che esprime ed elabora le opinioni della critica precedente), la Deledda descrive costantemente nelle sue opere un modello di vita patriarcale che si rifa alle sue esperienze nella nativa Sardegna: "una vita arcaica sentita quasi biblicamente, con le sue leggi della necessità della pena, dell'espiazione dopo il compimento del male" (Convegno 213). ${ }^{2}$

La scrittrice trovava questo modello nell' ambito della propria famiglia e rifletteva nei suoi libri la sua visione della vita e della società. Per esempio, in una lettera del 20 maggio 1894 a Giovanni De Nava, un ammiratore dei suoi scritti, la Deledda così parla di sé:

Appartengo ad una famiglia di quei principali sardi che io metto spesso nei miei racconti; gente bizzarra, tra il patriarcale e il selvaggio, che non appartiene né alla borghesia né ai popolo né alla nobiltà. Forma una casta da sé, e quando, come la mia famiglia, può disporre di un patrimonio più o meno vasto, ha tutta la prepotenza boriosa che, più d'ogni altra cosa, accusa l'origine spagnuola. (Grazia Deledda 29-30)

Sempre dalle lettere della Deledda risulta chiaro che, anche quando è diventata una scrittrice affermata a Roma, continua a rispettare i principi della famiglia patriarcale e a credere nella subordinazione della donna all'uomo. In una lettera del 17 gennaio 1905 scrive al signor De Laigue, Console Generale di Francia a Trieste:

Se fossi nata uomo sarei stato un solitario; sarei vissuto in un eremo fra le braccia immense della grande Natura ... . Donna, devo adattarmi a piegarmi, a vivere fra coloro che amandomi e proteggendomi completano la mia esistenza. Ed io sono felice così, forse più che non lo sarei stata se fossi nata uomo. Ho un marito bello e buono e due bambini graziosi che adoro. (Momigliano 87)

Pur accettando le regole ancestrali della famiglia patriarcale, e riaffermandone immancabilmente i valori, la Deledda tratta ripetutamente l'infrazione dei tabù sessuali e delle strutture sociali della famiglia. Come il Sapegno scrive in "Ricor- 
do di Grazia Deledda" del 1946, possiamo individuare nella scrittrice

un motivo inquieto, ardente, di abbandono passionale, di rivolta alle convenzioni stabilite e ai pregiudizi consacrati, tipicamente romantico e una tendenza invece rassegnata e stanca, per cui gli slanci ribelli, le passioni insorgenti con impeto incontenibile, le inquietudini anarchiche tornano poco per volta a incanalarsi nell'alveo di una consuetudine, di una norma prestabilita, di un ordine e di una legge. (Pagine 290-91)

Il Sapegno aggiunge che questi elementi della scrittrice provengono da due fonti diverse. Da una parte hanno una radice personale, in quanto riflettono "il suo spirito giovanile di rivolta contro le convenzioni della società borghese", spirito che poi si acqueta sempre di più nell'accettazione dell'ordine sociale e religioso stabilito (Pagine 291). (Non dobbiamo dimenticare che la Deledda si era ribellata al ristretto ambiente di Nuoro, in cui era nata e cresciuta, e lo aveva lasciato per seguire la sua vocazione di scrittrice e per sposare un lombardo.) Dall'altra tali elementi hanno una radice sociale, in quanto esprimono "i fermenti esplosivi ed anarchici di rivolta [della società] e gli stanchi ripiegamenti alla norma di una disperata fatalistica rassegnazione" (Pagine 294).

L'analisi del Sapegno inquadra in poche parole quella che è l'essenza dell'opera della Deledda e ne dà una visione dinamica e romantica. L'arte della Deledda consiste appunto nella tensione tra questi due elementi di ribellione ed esplosione delle passioni da una parte e di accettazione delle regole costituite dall'altra, col prevalere costante del secondo elemento. La scrittrice, secondo l'analisi del Sapegno, non è né la donna calata nel presente, che interpreta "la realtà della sua regione e della civiltà arcaica in crisi", come vorrebbe il Massaiu (111), né la donna retriva, chiusa nel passato e completamente estranea ai cambiamenti della società del tempo, come vorrebbe il Pellegrino (495). Abbiamo invece la visione romantica di una Deledda sospesa tra il vecchio e il nuovo, ancorata al passato e pur desiderosa di muoversi verso il futuro.

Altro tema importante da affrontare è il modo in cui la Deledda trasfigura i suoi personaggi liricamente, collegandoli alla natura e trasformandoli in creature mitiche. Pur derivando la visione della famiglia da esperienze della sua vita e pur documentandosi sugli usi e costumi dei Sardi (si veda il suo libro sulle Tradizioni popolari di Nuoro in Sardegna), la scrittrice fa lievitare questo materiale attraverso la forza della sua immaginazione e trasforma il dato di osservazione in elemento lirico. Già nel 1923 il Pancrazi dichiara che la Sardegna della Deledda "fu sempre un rifugio fantastico della sua poesia, una creazione del suo sentimento e dell'arte sua. La Sardegna reale fu soltanto lo schema, il pretesto di quest'altra Sardegna poetica e personale della Deledda" (90). Nel discorso ufficiale alla consegna del premio Nobel alla Deledda nel 1927, Henrik Schuck paragona i personaggi della Deledda con quelli di Omero e della Bibbia e ne nota la loro affinità col paesaggio: "Si direbbe quasi che gli uomini appaiono come piante germogliate dal suolo stesso della Sardegna. La maggior parte sono gente del popolo, 
creature semplici, con un modo di pensare e di sentire che ha qualcosa della grandiosità della natura sarda" (106). E il Sapegno, nella sua prefazione del 1971 all'edizione mondadoriana dei romanzi e delle novelle della Deledda, osserva che nella scrittrice "quella scelta di ambienti e di temi conformi era . . . la proiezione mitica di un ansioso ed acceso fervore, l'inquietudine di un'adolescenza solitaria" (xvii-xviii).

Nell' analisi del racconto "Battesimi" intendo mettere in rilievo tutte le caratteristiche dell'arte deleddiana qui sopra discusse: la sua presentazione dei personaggi come eroi mitici che vivono in contatto con la natura e sembrano essi stessi forze della natura, la sua visione arcaica della famiglia con le sue leggi e i suoi rituali che risalgono a tempi remoti, la ribellione appassionata di alcuni membri della famiglia contro l'ordine costituito e l'inevitabile trionfo della volontà del capo della famiglia a garantire la continuità della società patriarcale. Di particolare interesse nel racconto è il fatto che tramite la ribellione della madre e della figlia la posizione della donna, sottomessa all'uomo, venga messa in causa e che due riti fondamentali della famiglia, il battesimo e il matrimonio, vengano visti come strumenti di oppressione da parte dell'uomo, che nega ogni libertà alla donna. In questo caso intendo dimostrare come la ribellione delle donne della famiglia e la loro implicita aspirazione ad una diversa struttura famigliare rappresenti una forza destabilizzante nella vicenda, che tiene il lettore sospeso e lo costringe a leggere il racconto in due modi diversi allo stesso tempo.

$\mathrm{Nel}$ corso della mia analisi mi appoggerd alla teoria dei campi semantici com'è definita da Silvana Ghiazza nel suo articolo "Semantica della morte nella poesia del Foscolo". Secondo la Ghiazza potremmo dire che il campo semantico

a lo spazio entro cui si organizzano, in rapporti vicendevoli di forza e per associazioni semantiche di natura linguistica ed extralinguistica, lessemi appartenenti a categorie grammaticali diverse, tutti ruotanti attorno ad un concetto centrale, il quale si definisce e si articola proprio in virtú del loro vario e dinamico inter-agire. All'interno di questo spazio e solo in esso i singoli lessemi rivelano tutto il loro spessore semantico, in una stretta interdipendenza fatta di opposizioni e di richiami; attraverso il rifrangersi dell'uno sull'altro essi vengono definiti e nello stesso tempo potenziati e arricchiti nella loro estensione e intensità di significato; e dunque lo spostamento, l'eliminazione o l'inserimento di uno o piú di loro, ogni cambiamento rilevante insomma, all'interno del campo, si riflette necessariamente su tutti gli altri elementi, modificando non solo lo spessore semantico di ciascuno, ma anche quello dell'arcilessema, modificando il campo stesso nel suo complesso. $(371-72)$

Mi serviro di questa definizione in senso lato, identificando il concetto centrale del racconto e indicando come tutte le altre immagini e situazioni si sviluppano intorno a questo concetto o per somiglianza o per opposizione. Dimostrero, inoltre, come questi movimenti e tensioni si traducano nella mente del lettore in messaggi ambigui, inquietanti e molto moderni. Nel corso della mia analisi farò vedere come intorno all' arcilessema "acqua" (cioè il concetto centrale "il cui conte- 
nuto è identico con il contenuto dell'intero campo lessicale" [Geckeler 15]) ruotino dei sinonimi di acqua: "bufera", "pioggia potente", "tempaccio", "sogno tempestoso", "lavacro del battesimo", "onda schiumosa", "pianto". E intorno all'arcilessema opposto ad "acqua", cioè "fuoco", inteso nei suoi attributi di luce e colore, ruotino i sinonimi di fuoco: "lampi", "fiammeggiassero", "folgore", "fulgore", "incendio" e i colori "rossastre", "rosa", "oro" e "argento". ${ }^{3}$ Mostrerò, inoltre, come tali lessemi ricevano contemporaneamente una valutazione etica opposta da parte dei diversi personaggi del racconto e del narratore stesso, cioè simbolizzino situazioni e sentimenti contrastanti e creino quindi un senso di ambiguità in chi legge.

Il racconto è narrato al passato e ha luogo tra i contadini, in un posto non ben definito, e in un tempo non chiaramente fissato, ma certo non lontano dal presente (la gente va in giro in bicicletta). La trama del racconto è molto semplice. In una notte di tempesta all'inizio della primavera, una giovane sposa bussa alla porta dei genitori e cerca rifugio dalla brutalità del marito. Il padre è inflessibile di fronte alle suppliche della moglie e della figlia e riconduce quest'ultima dal suo sposo, in nome delle leggi ataviche della società contadina, che esigono che la donna si sottometta ciecamente al volere del capofamiglia e rinunci completamente alla propria individualità.

Il racconto "Battesimi" è diviso dalla Deledda in cinque parti, chiaramente demarcate da spazi vuoti nella pagina, e si svolge tramite un susseguirsi di scene e di immagini ricche di significato simbolico. Nella prima parte è descritta una tempesta e passiamo dal paesaggio esterno al colloquio serale di due coniugi nella loro casa. La seconda parte consta di un flashback della moglie che ripensa alla cerimonia del battesimo di sua figlia. La terza parte descrive le emozioni dei due coniugi che sentono un viandante misterioso (che si rivelerà poi essere la figlia) bussare alla porta durante la notte. La quarta parte, la più lunga e drammatica, segna lo scoppio della catastrofe, cioè il conflitto tra il padre e le donne della famiglia e la risoluzione di tale conflitto secondo le tradizioni contadine. Alla fine di questa parte la cerimonia del battesimo viene rivissuta di nuovo dalla moglie in un flashback molto piu breve. La quinta parte ci riporta al paesaggio esterno e conclude con i sentimenti degli sposi che si riconciliano e con quelli del padre di famiglia, che ristabilisce l'ordine e in questo modo si sente vicino ai grandi legislatori del passato.

La vicenda è narrata da un narratore onnisciente, che ci scopre gli intimi pensieri dei suoi personaggi. Il tempo della storia, che dura per un periodo molto breve, corrisponde al tempo del racconto, eccezion fatta per i due flashback della moglie che ripensa al battesimo della figlia. Il titolo del racconto "Battesimi", pieno di suggestioni mitico-religiose, prepara il lettore ad esaminare il significato del rito che segna la purificazione dell'individuo dal peccato originale e la sua entrata ufficiale nel mondo cristiano. L'uso del plurale e la mancanza dell'articolo determinativo (cioè "Battesimi" e non "Il battesimo") indica che tale rito avviene in un contesto particolare e per un numero limitato di individui. Visual- 
mente il titolo evoca una cerimonia di purificazione spirituale tramite il contatto con l'acqua e ci introduce al concetto centrale "acqua" che domina il campo semantico del racconto.

La nozione dell'acqua come elemento sacro e vivificante è ripresa nella descrizione della tempesta all'inizio del racconto:

Dio volendo, dopo una lunga siccità invernale che pietrificava la terra e le piante, era tornata una classica notte di vento, di pioggia potente, di lampi e di tuoni. La casa tremava tutta, ma pareva per gioia, per accompagnare lo sfregamento di mani del proprietario, per rispondere al fremito fuori dei campi che si ubbriacavano di acqua. (71)

L'arrivo della primavera è qui visto come benedizione del cielo che riporta in vita la terra pietrificata dall'inverno, sciogliendola con la pioggia. L'acqua qui non è tanto elemento purificatore secondo il rito cristiano quanto forza fecondatrice della natura secondo antichi riti pagani, liquido inebriante che libera forze e sentimenti repressi durante l'inverno. Al fremito orgiastico dei campi, che si ubriacano d'acqua, quasi spiriti della natura in un corteo dionisiaco, corrisponde il tremito di gioia della casa, che accompagna il gesto di soddisfazione del proprietario che si sfrega le mani al pensiero dei campi resi fertili dalla pioggia.

Il paragrafo comincia e finisce con la visione della pioggia nella natura e procede con movimento circolare. Passiamo dall'alto (Dio e il cielo piovoso) al basso (i campi e la casa) e dal paesaggio esterno all'interno della casa, per terminare di nuovo col paesaggio esterno sotto la pioggia, intesa in senso rituale. Da notare che la casa è personificata come creatura umile e sottomessa che trema di gioia al piacere del suo signore. Cioè dal rapporto tra Dio e i fenomeni naturali e da quello tra la pioggia e la terra inaridita, si passa al rapporto tra il marito/padrone e la donna/casa, per ritomare poi ai rapporti iniziali.

Tale passaggio dai fenomeni della natura ai rapporti umani diventa esplicito nei paragrafi seguenti. Il marito, che si sta spogliando per andare a letto, comunica i suoi pensieri alla moglie già rannicchiata nel letto, che ringrazia Dio della pioggia. La donna è "tremante e felice", come la casa nel paragrafo precedente, ma ha anche "un senso di paura in fondo all'anima". Cioè la donna si differenzia dalla casa nel suo rapporto ambivalente verso il marito. Lei apre gli occhi quando il marito entra nel letto, e lo vede come una divinità della natura, mezzo nudo, rosso e nero, illuminato dai lampi e come fiammeggiante:

[La moglie] pregava e solo quando il marito, mezzo nudo, con le coscie e le gambe rossastre chiazzate di ricciolini neri, i grandi piedi gelati, fece scricchiolare col suo corpo pesante la stoppia del saccone, aprì gli occhi e le parve, per il riflesso della finestra illuminata dai lampi, che fiammeggiassero anch'essi. Poi si ricoprì, e la voce del marito le arrivò di lontano, quasi echeggiante sotto le coltri di piuma. Era una voce cattiva, anzi beatamente crudele. (71-72)

Nel campo semantico dell'acqua viene ora introdotta l'idea opposta del fuoco. 
I tuoni e i lampi, entrati nella narrativa tramite la descrizione del temporale, si concretano nella rappresentazione di una creatura in parte umana e in parte divina: la figura del marito. Questi, infatti, evoca le divinità infernali, sia per il colore rosso e nero delle sue gambe, sia per le fiamme che lo circondano. Se a livello mitico il personaggio del marito è un dio del fuoco e della notte, a livello di vita sociale e famigliare è un essere dominatore e crudele. Il tuono esterno diventa il suono della sua voce cattiva, da cui la moglie tenta invano di ripararsi.

L'uomo espone a lungo la sua visione della vita alla moglie, che lo ascolta in silenzio e solo a un certo punto dice: "Così sia", quasi che i comandi di lui esprimessero la volontà di Dio. Il marito gode crudelmente delle sofferenze dei viandanti che si trovano sperduti nei campi senza alcun riparo dalla pioggia e fa alla moglie un quadro della società e della vita famigliare basata sull'autorità del capofamiglia. Secondo lui l'uomo deve controllare e reprimere la donna, allo stesso modo in cui il contadino coltiva la terra e alleva le bestie:

... voi donne vedete tutto facile, tutto semplice, mentre la vita è una cosa difficile. La vita, - rincalzò con voce grossa, sebbene la moglie non fiatasse, - è come tutte le aitre cose; come le bestie, come le piante, come le erbe: bisogna tenerla a freno, potarla, falciarla: o, se ti pare meglio, è come la barba, che se tu non te la radi ogni otto giorni, con fastidio, con pericolo di tagliarti, t'invade il viso e ti fa scacciare dal consorzio degli uomini civili. (72-73).

Il marito vede la moglie e la figlia come esseri ingenui, inesperti e impulsivi, che devono piegarsi alle decisioni degli uomini della famiglia. In questo caso il simbolismo dell'acqua, intesa come violenza ai viandanti sperduti, ci prepara a una visione della società basata sulla violenza fisica e psicologica fatta dall'uomo alla donna. L'acqua e il fuoco del temporale si traducono nell'atteggiamento brutale dell'uomo verso le donne della sua famiglia.

La seconda parte della storia ci scopre i pensieri della moglie, che in apparenza è passiva, silenziosa e completamente sottomessa al marito; in realtà ripensa alla condizione umiliata e offesa della donna, simbolicamente rappresentata dal battesimo della figlia. La donna ricorda la violenta reazione della sua piccola "muta, cieca, informe, eppure già bella, sensibile, vibrante di vita", al sentire il "Credo. Rinunzio" del battesimo pronunziato dal prete e dagli astanti: "[La piccola] agita i pugni con una forza che fa sorridere la madre; e smorfie di protesta, di noia, di disgusto le smuovono il visino come se un sogno tempestoso le agiti l'anima ancora addormentata" (73).

La tempesta del paesaggio iniziale diviene qui tempesta di emozioni della neonata che protesta di fronte alle imposizioni della società in cui sta per entrare ufficialmente. Vicino alla figura del sacerdote domina quella del padre "grezza ... cogli occhi neri, la barba nera, le sopracciglia che sembrano baffi ....". Nel "quadro gaio e dorato del battesimo" lui "sorveglia tanto la madre quanto la figlia" e introduce una nota di terrore e di lutto (74). La figura cupa e minacciosa del padre contrasta con la vitalità della neonata a cui si accompagna una connotazione 
di luce e di gaiezza. Quella che dovrebbe essere una cerimonia di purificazione e di rinascita per la bambina, diviene in questo modo una cerimonia di rinuncia alla propria individualità e di morte dell'anima. Il fatto, poi, che la scena del flashback sia narrata al presente, in contrasto col resto del racconto che è narrato al passato remoto o all'imperfetto, dà maggior rilievo a tale scena, perché ce la mostra non come un avvenimento già concluso, ma come qualcosa che si svolge sotto i nostri occhi, in un eterno presente.

La terza parte riprende l'immagine finale della scena precedente, colla figura minacciosa del padre, che sente picchiare alla porta durante la tempesta. Come già all'inizio del racconto, il paesaggio esterno si fonde coi sentimenti interni dei personaggi. Il fragore della bufera si traduce nel cipiglio del padre e i colpi alla porta, battuti da un misterioso viandante, sono echeggiati dal battito del suo cuore. In questo passo il marito viene umanizzato, sia pur per breve tempo, e la sua figura non è monolitica come prima. Lui prova ansietà ed esitazione, prevedendo che si tratti della figlia alla porta. Il cuore gli batte e le dita gli tremano mentre si abbottona il vestito.

I pensieri ansiosi e tempestosi del marito si estendono anche alla moglie, che si sveglia preoccupata e ascolta quello che succede dalla camera da letto. La Deledda descrive la moglie che emerge da sotto le coltri con delle immagini acquatiche ed aeree, che contrastano nettamente colle cupe visioni della tempesta:

La sua testa d'oro e d'argento affiorò sui guanciali e le coltri, come uscendo da un'onda schiumosa: gli occhi grandi e azzurri di bambina spaventata cercarono invano quelli del marito. Egli già lasciava la camera, portandosi via il lume. - Signore, Signore, - invocò la donna, e stette ad ascoltare nel caos della bufera, del letto scoperto, dell'agitazione del suo cuore. (75)

In questo caso la tempesta di rabbia e di apprensione che sconvolge il cuore del marito all'inizio della scena e che diviene tempesta di ansia e terrore per la moglie alla fine della scena, contrasta colla visione dell'onda schiumosa dei guanciali da cui emerge la testa d'oro e d'argento della donna. Al mondo cupo, violento e tempestoso di una società patriarcale, dove l'uomo domina colla violenza le donne della sua famiglia, si oppone la visione luminosa e serena di una società matriarcale dove la donna emerge dall'acqua, come Afrodite dalla spuma del mare, e dove la primavera viene celebrata come pienezza di vita e non come mortificazione dell'anima. I colori dell'oro, dell'argento e dell'azzurro della donna, che pare emergere dall'acqua, suggeriscono anche la purezza della neonata, lavata dall'acqua del battesimo ed emergente a nuova luce e mettono in contrasto questa scena con quella del battesimo della figlia, descritto nella parte precedente. In questa visione serena gli elementi dell'acqua e del fuoco/luce (l'oro e l'argento) celebrano la femminilità invece di mortificarla.

La quarta parte, la più lunga e drammatica, ci rappresenta lo scoppio dei conflitti psicologici già accennati prima e l'apparente risoluzione di tali conflitti. I moti psicologici dei personaggi sono messi in evidenza dallo sviluppo della te- 
matica dell'acqua, vista come violenza, rinunzia e morte dell'anima, una tematica già messa in rilievo nelle parti precedenti. La moglie che ascolta ansiosa dalla sua camera, tendendo le orecchie, è ferita dal rumore della tempesta:

... le pareva che la pioggia fredda e moribonda le penetrasse fino al cuore. E non osava muoversi oltre, con un senso di terrore panico. Ma un grido salì dalla strada, fece tremare la camera e la illuminó col boato e la luce della folgore. - Mamma! - La donna si precipitò dal letto, si precipitò per le scale, fu nell'ingresso. In camicia, scarmigliata, pareva fuggisse da un incendio. (75-76)

Come già nella prima parte, anche qui l'immagine della tempesta porta con sé quella dei tuoni e dei lampi che si concretano in voci e gesti dei personaggi: in questo caso nel grido disperato della figlia e nella corsa precipitosa della madre verso la sua creatura. Particolarmente interessante il movimento della madre, che sembra fuggire da un incendio, ma che in realtà corre verso il fuoco delle passioni contrastanti tra suo marito e sua figlia. Mentre nella prima parte dio del fuoco era l'uomo, qui le immagini di fuoco e di luce folgorante appartengono alla donna, che non subisce più passivamente la volontà dell'uomo, ma che lotta per riaffermare l'antico legame tra madre e figlia. Demetra, l'antica dea della terra, vuole ricongiungersi alla figlia Persefone. Tale mito matriarcale, tuttavia, non può attuarsi nel contesto di una società patriarcale, dove l'uomo ha diritto assoluto sulla donna e dove la figlia sposata appartiene completamente al marito.

La madre angosciata è costretta ad assistere allo scontro tra padre e figlia, col padre che sbarra con le sue braccia la porta alla figlia grondante e le impedisce di rifugiarsi nella casa dei genitori:

Il marito stava sulla porta appena dischiusa, lapidato dalla pioggia, e parlava con la persona alla quale impediva di entrare. Appena si accorse che la moglie era alle sue spalle si volse di scatto, livido, col viso bagnato come di un sudore di lotta, e aprì di più la porta, ma sbarrandola con la stanga delle braccia nerborute: ed ella vide la figura che già le stava nelle pupille smarrite. La figlia era li, pallida e grondante d'acqua come un'annegata, e invano domandava di entrare. (76)

La madre diviene progressivamente conscia di quello che voglia dire essere donna. Al grido della figlia, simile a una folgore nell'oscurità, segue la visione rivelatrice del padre lapidato dall'acqua della tempesta, bagnato dal sudore della lotta che deve fare sia con la volontà disperata della figlia sia con i suoi stessi sentimenti di tenerezza verso di lei. E, al di là delle braccia nerborute del padre, che impediscono alla figlia di entrare e di ricongiungersi alla madre, la donna riconosce nella ragazza grondante d'acqua, quell'immagine della sua bambina, che ha gia fissa nel cuore e in cui rispecchia la sua stessa condizione di creatura umiliata e sottomessa.

Invano le due donne cercano di piegare la volontà del padre con preghiere e spiegazioni. L'uomo "aveva ripreso la sua aria di beffa crudele e pareva prendere gusto alla lotta" (76). Lui rimane inflessibile di fronte alle lacrime della moglie, 
che vorrebbe almeno che la figlia si asciugasse, e alle suppliche della figlia, che non sopporta di rimanere con il marito e implora la madre, "per amore di Cristo", di prenderla con sé. Anzi, il padre risponde brutalmente alle due donne, minacciando di picchiarle e canzonandole, quasi fossero due bambine capricciose e disubbidienti. Le voci, quella beffarda del padre e quella disperata della figlia, "risonavano assieme, come in un duetto tragicomico, accompagnato dal coro della bufera" (77). Alle parole della figlia, che invoca la madre, come in una sacra rappresentazione, si sovrappongono le parole insultanti del padre, che pensa solo a non svegliare i vicini e a rispettare le convenzioni del paese. Il mondo del mito e della tragedia, rappresentato dalla madre e dalla figlia, si abbassa al livello comico e volgare del padre.

Alla fine di questa lotta, la madre è costretta a cedere e ad ubbidire alla volontà del marito, ma da questo contrasto le deriva una coscienza più profonda della sua condizione:

La donna si passò una mano sul viso, come strappandosi un velo; ancora una volta rivedeva la sua piccola bambina, vestita di rosa dal fulgore di un lampo, aspersa dal lavacro del battesimo. - Credo. Rinunzio. - E ando a prendere una dopo l'altra le biciclette e il cappotto del marito. Porto anche uno scialle, per la piccola; ma fu rifiutato. La piccola era già ben coperta dal suo scialle di pioggia e di obbedienza al destino. (77)

Questo flashback della madre, che riprende la lunga descrizione del battesimo nella seconda parte del racconto, acquista il senso di un'ulteriore rivelazione (indicata dall'immagine tradizionale del velo squarciato). Il ricordo della bambina piena di vita, rosea nel fulgore di un lampo e purificata dall'acqua del battesimo (immagini in cui acqua e fuoco hanno un significato vivificante per la neonata) che deve rinunciare alla propria identità e sottoporsi alla volontà del padre ("Credo. Rinunzio") si fonde colla visione presente della figlia, illuminata dalla luce dei lampi, e costretta di nuovo a sottomettersi alla volontà del padre e a quella del marito. Il matrimonio diventa un'estensione del battesimo, visto come rito di violenza e di morte. Cioè il significato originariamente positivo e vitale dell'acqua e del fuoco, presente nel battesimo e nella descrizione iniziale della pioggia, cede il posto al significato negativo dell'acqua della tempesta e del fuoco dei lampi, che rappresentaiio la dura legge della società contadina. La tempesta percuote la donna senza pietà e diventa uno scialle con cui essa deve coprirsi, come se fosse in chiesa, e il fuoco dei lampi è implicitamente un'estensione della figura tirannica del padre, come abbiamo già visto nella prima parte.

La quinta e ultima parte del racconto si svolge tutta a livello mitico. Coll'allontanarsi del padre che riaccompagna la figlia dal marito, la furia degli elementi si placa:

... la tempesta parve fare ala come un popolo in tumulto che si calma al passaggio di un viatico, a quei due che l'attraversavano con la forza del loro dolore . . . . Ma quando furono nella casa degli sposi, e questi si riconciliarono, un po' per amore, 
molto perché sentirono il dominio inesorabile che oramai li teneva, egli, senza volerlo, senza neppure saperlo, si sentì vicino ai grandi primi uomini che con la violenza avevano creato le leggi per i loro simili. (78)

Qui il padre non è piu l'essere crudele e sinistro delle parti precedenti del racconto, che gode nel tormentare le donne della famiglia, ma diviene l'eroe civilizzatore che deve usare la violenza per reprimere in se stesso e nella famiglia "ogni istinto di ripiegamento e di riposo" (78). La conclusione della storia sembra, dunque, dare ragione al padre e sancirne il comportamento violento, presentato come necessario al mantenimento delle leggi sociali. Rimangono, tuttavia, alcuni problemi da risolvere, creati dalle immagini stesse del testo e dalla disposizione di tali immagini, che ci portano a una diversa interpretazione del racconto.

L'immagine centrale dell'acqua e quella del suo opposto, il fuoco, hanno significati ambigui e contrastanti che sembrano suggerire due letture opposte. La tempesta di primavera, con i suoi tuoni e lampi, e il battesimo, con il suo fulgore e la sua acqua purificante, hanno da un lato il significato di fertilità, rinascita e sviluppo sociale dell'uomo, dall'altro il significato di violenza e brutalità dell'uomo verso la donna, e di perdita d'identità della donna sottomessa all'uomo. I fenomeni naturali e i riti sociali vengono dunque a coincidere nel corso del racconto.

La vicenda procede in modo lineare dall'inizio alla fine del racconto e segna il trionfo dei valori del padre-padrone sui desideri della moglie e della figlia. Tali valori sono presentati come un trionfo della morte sulla vita. La pioggia alla fine fa ala al padre e alla figlia come una folla che si apre al passaggio del viatico. E la donna che viene sacrificata ai voleri dell'uomo. In contrasto con questo sviluppo lineare, l'immagine del battesimo, già introdotta dal titolo del racconto, si insinua due volte nel racconto e crea un movimento centripeto, che contrappone i valori della donna alla volontà tirannica dell'uomo. Il fulgore roseo della piccola al battesimo, il grido della figlia ormai adulta che scoppia nella mente della madre come una folgore, introducono un modo ben diverso di vivere e di comunicare, un modo più istintivo, più poetico, più bello e più giusto. La visione della madre, che emerge tutta oro e argento dalla spuma delle coltri, ci riporta a visioni pagane e cristiane di un mondo rinato in primavera e di un vero battesimo, inteso come pienezza di vita (si ripensi al paradiso terrestre di Dante).

L'alternarsi e il sovrapporsi di questi diversi significati dell'acqua e del fuoco, e i movimenti delle immagini in senso diverso creano una forte tensione e ambiguità nel racconto. Il lettore, alla fine, rimane sospeso tra i significati contrastanti dello stesso campo semantico ed è costretto a leggere il racconto in due modi diversi allo stesso tempo: assumendo ora il punto di vista dell'uomo ora quello della donna, senza poterne risolvere l'innata contraddizione. In tal modo una situazione conflittuale, che probabilmente riflette sia l'ambivalenza psicologica della Deledda stessa, sia le tensioni della società contemporanea, trova la sua espressione nel simbolismo e nella struttura stessa del racconto. 


\section{NOTE}

1 Questo racconto è incluso con un breve commento nell'antologia di Pickering-Iazzi e Baldassaro (27-31). Gli autori mettono in rilievo il rapporto tra il mondo contadino descritto dalla Deledda e le norme della società patriarcale imposte dal govemo fascista negli anni venti, in cui è scritto il racconto.

2 La società patemalistica nell'opera della Deledda è trattata anche in Piromalli (Grazia Deledda 9-30).

3 Per una spiegazione dell'arcilessema, si veda anche Coseriu 44-45.

\section{OPERE CITATE}

Coseriu, Eugenio. "Vers une typologie des champs lexicaux". Cahiers de Lexicologie 27 (1976): 3051.

Deledda, Grazia. La casa del poeta. Milano: Treves, 1930.

. Romanzi e novelle. Ed. Natalino Sapegno. Milano: Mondadori (I Meridiani), 1971.

. Tradizioni popolari di Nuoro in Sardegna. Roma: Forzani, 1894.

Geckeler, Horst. "Major Aspects of the Lexematics of the Tubingen School of Semantics". Understanding the Lexicon. Ed. Werner Hullen and Rainer Schulze. Tubingen: Max Niemeyer Verlag, 1988. 11-22.

Ghiazza, Silvana. "Semantica della morte nella poesia del Foscolo". Quaderni di semantica 3.2 (1982): 369-405.

Grazia Deledda. Biografia e Romanzo. Roma: Istituto della Enciclopedia Italiana, 1987.

Massaiu, Mario. La Sardegna di Grazia Deledda. Milano: CELUC, 1972.

Momigliano, Attilio. Ultimi studi. Firenze: La Nuova Italia, 1954.

Pancrazi, Pietro. Venti uomini, un satiro e un burattino. Firenze: Vallecchi, 1923.

Pellegrino, Angelo. "Deledda, Grazia". Dizionario Biografico degli Italiani. Vol. 36. Roma: Istituto della Enciclopedia Italiana, 1988. 491-96.

Pickering-Iazzi, Robin, e Lawrence Baldassaro. In terza pagina. New York: Holt, Rinehart and Winston, Inc., 1989.

Piromalli, Antoniu. "Cultura e società nei primi romanzi di Grazia Deledda". Convegno Nazionale di Studi Deleddiani. Cagliari: Fossataro, 1972. 211-26.

. Grazia Deledda. Firenze: La Nuova Italia, 1968.

Sapegno, Natalino. Pagine di storia letteraria. Palermo: Manfredi, 1960. . Prefazione. Deledda, Romanzi e novelle xi-xxiii.

Schuck, Henrik. "Discorso ufficiale alla consegna del Nobel". Grazia Deledda. 97-106. 